\title{
COCINA NACIONAL, PROCESOS IDENTITÁRIOS Y RETOS DE SOBERANÍA: LAS RECETAS CULINARIAS CONSTRUYENDO MOZAMBIQUE ${ }^{1}$
}

\author{
NATIONAL KITCHEN, IDENTITY PROCESSES AND CHALLENGES OF \\ SOVEREIGNTY: CULINARY RECIPES BUILDING MOZAMBIQUE
}

\author{
Maria Paula Meneses ${ }^{2}$
}

\section{RESUMEN}

¿Puede el conocimiento producidos por mujeres, en forma de recetas, parte de la red del Océano Índico, promover una interpretación más amplia acerca de los contactos e historia, más allá del imaginario colonial? Asiente en dados de archivo e investigación etnográfica, este trabajo tiene como objetivo comprender cómo la comida puede personificar contactos geográficos y culturales, procesos de identidad que desafían las representaciones nacionales vigentes sobre la soberanía alimentaria. Como el texto problematiza, los alimentos siguen siendo símbolos particularmente potentes de la identidad personal y de grupo, formando uno de los fundamentos de la individualidad y de los sentidos de pertenencia común a un grupo más grande (cosmopolita, a menudo). Lo que es mucho menos comprendido es como un pilar tan estable de la identidad también puede ser tan fluido y cambiante; cómo se pueden mantener los límites, aparentemente insuperables, entre las prácticas y los hábitos dietéticos específicos de cada grupo, mientras las dietas, recetas y cocina están en constante flujo. Este texto interroga el colonialismo desde una perspectiva de la historia subalterna, buscando analizar los contactos coloniales a través de los alimentos, en un amplio contexto global. Intentaré entretejer episodios históricos dentro de una discusión más amplia - de presentar la dinámica de los contactos tras la participación de muchos grupos diferentes de personas, a menudo abrazando diferentes versiones del concepto de soberanía alimentaria. En un segundo momento exploraré las conexiones entre alimentos generadas por los encuentros coloniales, como forma de análisis de los efectos de la colonización y de los procesos migratorios, elementos clave para analizar la soberanía alimentaria como parte del proceso de afirmación cultural y soberanía política.

Palabras-Clave: cocina nacional, procesos identitários, Mozambique

\footnotetext{
${ }^{1}$ Este artículo fue elaborado con base en investigaciones realizadas no ámbito del proyecto "BLEND - Desejo, Miscigenação e Violência: o presente e o passado da Guerra Colonial Portuguesa” (PTDC / CVI-ANT / 6100 / 2014 - POCI-01-0145-FEDER-016859) financiado por la Fundação para a Ciência e Tecnología (FCT) de Portugal, con fondos nacionales y cofinanciado por FEDER a través del Programa Operacional Competitividade e Inovação COMPETE 2020, que coordino.

${ }^{2}$ Investigadora coordenadora do Centro de Estudos Sociais da Universidade de Coimbra, integrando o núcleo de estudos sobre Democracia, Cidadania e Direito (DECIDe). Doutora em antropologia pela Universidade de Rutgers (EUA) e Mestre em História pela Universidade de S. Petersburgo (Rússia). Lecciona em vários programas de doutoramento do CES, sendo co-coordenadora do programa de doutoramento em 'Póscolonialismos e cidadania global'
} 


\title{
govista \\ Debates Insubmissos
}

\begin{abstract}
Can women's knowledge, in the form of recipes, part of the Indian Ocean network, produce a broader image about contacts, history, beyond the predominant colonial imaginary? Based upon archival data and ethnographic research, this paper aims to understand how food can embody geographical and cultural contacts, identity process that challenge the prevailing national representations on food sovereignity. As I will seek to discuss, food remains a particularly potent symbol of personal and group identity, forming one of the foundations of both individuality and of a sense of common membership in larger, bounded (and quite often cosmopolitan) groups. What is much less well understood is how such a stable pillar of identity can also be so fluid and changeable; how the seemingly insurmountable boundaries between each group's unique dietary practices and habits can be maintained, while diets, recipes, and cuisines are in a constant state of flux. This text interrogates colonialism from a subaltern history perspective, and places colonial contacts through food in a broad global context. I will try to weave together historical snapshots with broader discussions - to present a lively picture of the dynamics of contacts, involving many different groups of people, often embracing different versions of the concept of food sovereignty. In a second moment, I will address the food connections brought about by colonial encounters, to broaden the debate on impacts of colonization and migratory processes, key elements to analyze food sovereignty as part of the process of cultural affirmation and political sovereignty.
\end{abstract}

Keywords: national kitchen, Identity processes, Mozambique

\section{INTRODUCCIÓN}

Los alimentos, especialmente los alimentos preparados, no sólo están estrechamente relacionados con el gusto / sabor y el hambre, sino, lo más importante, son también un criterio decisivo para la construcción de una identidad cultural. En otras palabras, los alimentos cocinados y las prácticas que se adjuntan a ellos proporcionan importantes criterios culturales que contribuyen a la asignación de una identidad cultural específica a una determinada parte de la sociedad. ${ }^{3}$

La idea de que las cosas, en este caso los alimentos, tienen vida social, o sea, tienen trayectorias que es importante estudiar, es propuesta por Arjun Appadurai (1991). Este autor plantea cuestiones importantes para el estudio de la circulación de los alimentos y de las recetas que los transforman en platos culinarios. Haciendo eco de la crítica de Foucault

\footnotetext{
${ }^{3}$ Utilizo el término 'cultural' no para clasificar, sino para describir prácticas relacionadas con la preparación y el consumo de alimentos, prácticas que las personas aprenden con sus familiares y sus comunidades, pero también de los 'otros' con quienes entran en contacto a través del comercio, de los violentos procesos de colonización, esclavización, etc.
} 
(1966), Appadurai señala las implicaciones del análisis eurocéntrico del mundo, análisis que (re)produce activamente la oposición entre las 'palabras' y las 'cosas'. Este enfoque, mientras establece que el mundo de las cosas es "inerte y mudo", defiende que este solo puede ser conocido "mediante las personas y sus palabras" (Appadurai, 1991, p. 19). Este abordaje estimula a ultrapasar la dicotomía entre las palabras que se mueven y las cosas, comprendidas como estáticas. La idea de estudiar las cosas en movimiento, circulando en diferentes contextos, sirve justamente para lograr entender los significados complejos de los usos y las formas que un determinado objeto (como los alimentos) toma durante su vida social. En este sentido, el reto es estudiar los conocimientos y las cosas en constante movimiento y fusión, dinamismos reveladores del contexto humano y social en que ocurren estos procesos (Ingold, 2011, p. 136).

El enfoque de este capítulo, que se basa en el estudio de los alimentos cocinados específicamente en el caril - es de estudiar la soberanía alimentaria en varios contextos de Mozambique como una estrategia de autoafirmación de las mujeres. El caril, y los saberes envueltos en su preparo, es utilizado para estudiar cómo se negocian espacios de reconocimiento que la colonialidad del saber y del ser insiste en no reconocer (MaldonadoTorres, 2007). ${ }^{4}$

La soberanía alimentaria, puesto brevemente, es el derecho de las comunidades a determinar su propia comida y los sistemas de producción de alimentos, defendiendo la autosuficiencia alimentar de la comunidad y/o autonomía cultural en relación con los alimentos. Esta propuesta representa un incontestable desafío a las tentativas de marginación económica y política de los campesinos y pequeños agricultores por las instituciones internacionales que gobiernan el comercio global de alimentos (LVC, La Via Campesina, 1996). ${ }^{5}$ Al problematizar las narrativas dominantes sobre las luchas por los alimentos en

\footnotetext{
${ }^{4}$ El caril remite a la salsa, con condimentos, que es uno de los platos de referencia en Mozambique, mientras el curry es un término genérico al polvo con varias especias distintivas, como la cúrcuma, comino, cilantro, fenogreco, pimienta, entre otras. La preparación del polvo de curry tiene su origen en Asia y es una de las herencias del imperio británico. El caril, como saber, ha sido apropiado por la cocina 'nacional' de varios lugares del mundo, con distintas comunidades reclamando su legitimidad sobre su origen (Leong-Salobir, 2011, p. 8).

${ }^{5}$ Vea se la definición de soberanía alimentaria en la Declaración de Nyéléni, producida en el marco del Foro para la Soberanía Alimentaria (2007, Mali), disponible en https://viacampesina.org/en/index.php/main-issues-
} 
Mozambique, este texto desafía varios de los enfoques metodológicos y epistémicos actuales, mostrando, desde ejemplos concretos, como la idea de seguridad alimentaria no habla acerca de dónde vienen los alimentos, quiénes lo produce, o las condiciones en las que se cultiva. Silenciando los millones de personas realmente responsables de la alimentación de Mozambique, el abordaje de la seguridad alimentaria permite a los exportadores de alimentos del Norte global argumentar que la mejor manera para los países considerados 'menos desarrollados' - como Mozambique - alcancen la seguridad alimentaria es subvencionar e importar alimentos baratos, recibirlos como 'ayuda alimentaria' en lugar de producirlo ellos mismos, o desarrollar regímenes de producción a gran escala de alimentos industrializados (Matavel et al., 2011).

Este es un texto sobre alimentos y recetas, sobre cocina y las pequeñas voces de mujeres, sobre el consumo y los sentidos de pertenencia. A partir del trabajo etnográfico (observación, entrevistas y conversaciones mantenidas en Mozambique desde 1998) y de archivo, busco discutir como la comida sigue siendo un símbolo particularmente potente de la identidad personal y colectiva, siendo uno de los fundamentos tanto de la individualidad como de la pertenencia a grupos más amplios (a menudo cosmopolitas). La comida, los platos preparados de las manos de las mujeres en Mozambique, de alguna manera sirven a reunir, o convocar, muchas de las relaciones necesarias para que la gente viva bien, gane su dignidad y pueda hacer planes para el futuro. En este sentido, la soberanía alimentaria es fundamental por problematizar, de un lado, los modelos de producción agrícola dominantes, y, por otro, por apuntar posibilidades de producción y consumo sostenibles, respetando la sociobiodiversidad, las prácticas agrícolas que designamos tradicionales (lo que incluye reconocer las semillas criollas, las recetas de su preparación, etc.).

El texto está organizado en tres partes. En la primera intento discutir cómo la historia colonial, de matriz eurocéntrica, impide una lectura más amplia de los productos y de su circulación. La expansión por el mundo de alimentos cultivados provenientes de todos los continentes es muy anterior al proceso que hoy es designado como globalización alimenticia

mainmenu-27/food-sovereignty-and-trade-mainmenu-38/262-declaration-of-nyi y la Declaración de los derechos de los y las campesinas/os (2009, Seúl), en el marco de La Via Campesina, disponible en https://viacampesina.net/downloads/PDF/EN-3.pdf. Ambos documentos accedidos el 2 de agosto de 2016. 
(Mintz, 2001, p. 32-33); inúmeros trabajos producidos en las últimas décadas apuntan a que estas conexiones han producido flujos globales cuya importancia es mucho más amplia que el fenómeno económico normalmente descrito como globalización económica (Inglis y Gimlin, 2009). Esta producción y circulación de alimentos, subalternizada por la lectura dominante sobre las culinarias del mundo, sugiere otras formas de ser y estar en el mundo, una situación que Boaventura de Sousa Santos describe como cosmopolitismo subalterno (2010, p. 29-31). La segunda parte explora, con un enfoque metodológico basado en la sociología de las emergencias (Santos, 2010), alternativas a la colonialidad del poder, del ser y del saber que continúan marcando el mundo contemporáneo. ¿Pueden pensar las mujeres de Mozambique más allá del imaginario colonial creado acerca de ellas? En las márgenes africanas del Índico, como en otros lugares, la comida encarna la historia, la geografía, los contactos culturales, de clase, de género y de identidad. Marcadas y marcando un reto a la tentativa de sumisión colonial, las voces subalternas simbolizan la lucha contra los intentos de dominación, trayendo al debate los saberes y los archivos que se han mantenido en silencio y silenciados, como explora la tercera parte de este texto. El desafío es sobre cómo descolonizar las bibliotecas y los archivos, superando las representaciones eurocéntricas resultantes del delirio colonial. Desde el reconocimiento de la alimentación como acción y como saber, intento abrir el debate sobre la sostenibilidad de los sistemas agroalimentares, entendidos desde la en el marco de la propuesta de las epistemologías del Sur avanzada por Boaventura de Sousa Santos (2014).

\section{MOZAMBIQUE: FLUJOS DE CONTACTOS Y EL RIESGO DE LA NARRATIVA EUROCÉNTRICA}

La comida es una gramática llena de sentidos. En ella se puede analizar quien produce y quien consume los alimentos que comemos, lo que coloca, desde el punto de vista político, cuestiones de soberanía alimentaria; pero también puede ser una gramática de clase, género o de marcación étnica asociado con limitaciones jerárquicas. Por ejemplo, la alta cocina 'global' se impone, con sus estrellas en la Guía Michelin, a la cocina de nuestros hogares, que se trasfigura en cocina tradicional o étnica. 
La circulación es una de las principales características de la humanidad; movimientos, contactos, tránsito de saberes, deseos y gustos dan forma a nuestras identidades. Paradójicamente la comida se basa en el mito del local, es decir, en un sentido de pertenencia situado. Cualquier receta culinaria tiene sus raíces: el contexto cultural que la ha producido, sus instrumentos y sus ingredientes propios; pero esto no significa que las recetas no se muevan libremente y no se adapten con fluidez a nuevas circunstancias locales. Es la química entre el conocimiento de su composición y de sus ingredientes, la relación entre los productos y entre éstos y los procesos y las herramientas de su transformación que hace que una receta sea lo que es, que adquiera su especificidad.

En este sentido, la comida, la preparación de los alimentos, al igual que cualquier otro elemento material culturalmente definido, fortalece a las comunidades, pero también genera especificidades culturales (Mintz e Du Bois, 2002, p. 109). La diversidad cultural africana, traducida a menudo en grupos étnicos, nace de la búsqueda de la diferencia y trabaja por contraste. Pero esta búsqueda por las especificidades, en los contextos del Índico africano, se ve obstaculizada por el hecho de que muchas continuidades marcan los grupos que habitan en la región, combinando simultáneamente, en el campo de la alimentación, el intercambio de técnicas e ingredientes entre las comunidades con rasgos y contenidos culturales específicos.

Una cocina étnica se presenta generalmente como asociada con una comunidad geográficamente y / o históricamente definida por los productos que consume y por las técnicas de preparación que utiliza, como es el caso de la cultura Swahili que se extiende desde la costa somalí hasta Mozambique (Rolingher, 2009). Pero cualquier etnia o nación son proyectos imaginados - y los platos culinarios asociados pueden ser imaginados, también. Una vez imaginadas, estas cocinas se pueden utilizar para realizar la idea de la identidad étnica o nacional. ${ }^{6}$

La creación de la representación de la comida nacional es uno de los elementos usados para añadir fuerza y coherencia conceptual a la idea de Mozambique, un país culturalmente muy diverso, con más de treinta grupos étnicos. ${ }^{7}$ La creación de una 'cocina nacional', como la situación en Mozambique revela, integra la invitación a una variedad de platos que se

\footnotetext{
${ }^{6}$ Como Priscilla Ferguson plantea (1998), es el discurso lo que define la cocina francesa.

${ }^{7}$ Ver, por ejemplo, Roletta (2004) y Sampaio (2007).
} 


\section{Revista $\bigcirc$ \\ Debates Insubmissos}

integren como parte de la visión de lo 'nacional'. En este sentido, la mención de un determinado plato como nacional anuncia sutilmente la nación (Appadurai, 1988). La 'cocina' étnica, nacional, en este sentido, no sólo se refiere a las recetas escritas, las descripciones de banquetes y fiestas, sino resulta también en una fijación normativa de ciertas prácticas culturales, que se transforman en códigos culturales fijos, símbolos de la mozambicanidad. En el contexto de la modernidad de matriz eurocéntrica, el proceso de nacionalización de la culinaria se produce mediante el establecimiento, por escrito, de los discursos sobre la nación, algunos de los cuales son recetas (Ferguson, 1998, p. 600). Y el poder de la palabra escrita, llena de imaginarios coloniales, aún permanece marcada en la "biblioteca colonial" (Mudimbe, 1988, p. 208).

Este legado colonial espinoso permanece arraigado en muchas referencias contemporáneas sobre el continente africano, con profundas implicaciones para la idea de la historia y las teorías de significado (Comaroff y Comaroff, 2010, p. 5). Sin embargo, hay que destacar que el desconocimiento de otras historias, transmitidas a menudo por vía oral, ha dado lugar a la presentación en varios libros sobre la cocina en Mozambique como algo que es exclusivamente el resultado de los encuentros ocurridos por ocasión de la presencia de Portugal, lo que silencia y esconde la diversidad y complejidades de los encuentros que los platos y las historias recogidas en la cocina pueden revelar. ${ }^{8}$ Por ejemplo, el enfoque en la explicación del 'comercio de especias' como una de las razones para crear imperios planetarios (Konetzke, 1946, p. 10), impide la comprensión de los circuitos comerciales más amplios que conformaban anteriormente y siguen conformando el Océano Índico, limitando el conocimiento sobre los intercambios y las fusiones de sabores (Sheriff, 1987; Meneses, 2009a, 2016).

En paralelo, la supuesta superioridad de la escritura como un espacio / tiempo de codificación cultural deja de lado muchos saberes, ya sea sobre la circulación de los productos, ya sea sobre las adaptaciones de los mismos para la preparación de diversos platos. En Mozambique el caril - la salsa hecha a base de pescado, carne, verduras o mariscos silenciosamente fue establecido como uno de los platos nacionales (Meneses, 2009a). Sin

\footnotetext{
${ }^{8}$ Véase, por ejemplo, el libro de Cherie Hamilton (2008).
} 


\section{Revista \\ Debates Insubmissos}

embargo, la diversidad del tejido social que constituye el Mozambique contemporáneo pone de manifiesto la presencia de una herencia culinaria diversa, reveladora de diferenciaciones y fusiones, resultado del violento encuentro colonial, de conflictos étnicos, regionales, de género y de la diferenciación de clases. La proyección del caril de camarones - una de las variantes más sofisticadas y caras - como uno de los platos nacionales de referencia elimina la diversidad de "cariles" que existen en el país, mientras que no tiene en cuenta las características regionales (dependiendo de los productos básicos y la capacidad financiera de las familias, entre otros). En otras palabras, el caril de camarones es un ejemplo de un plato que se convierte en un poderoso símbolo de representación nacional, ya sea en el país o en el exterior. ${ }^{9}$ Pero aun siendo un plato nacional, la presencia del caril de camarones en el mercado mundial hegemónico se encubre cuando se silencian los saberes acerca de las circulaciones de los productos que entra en su preparación y de los conocimientos asociados a los mismos. La tendencia, desde una perspectiva basada en los referentes de la meta-narrativa global eurocéntrica, de representar las comunidades que preparan este plato como nacionales $\mathrm{y} / \mathrm{p}$ locales, viene produciendo una densa descripción 'particularizada' de sus culturas; esta descripción hace caso omiso de importantes similitudes en las formas como los saberes y los sabores son hechos y rehechos continuamente en el mundo.

En realidad, platos híbridos, como los "caris" preparados en Mozambique, son únicos; distintos y diferentes de las prácticas alimenticias de Portugal, Asia (especialmente Goa) o Américas; al mismo tiempo incorporan componentes de la dieta de las tradiciones culinarias goesas, portuguesas, incorporando también ingredientes y prácticas indígenas de Mozambique. El caril es confeccionado de maneras muy diferentes y en momentos de celebración se consume asociado con varias otras especialidades, que incluyen, entre otros, las apas $^{10}$ y los achares. ${ }^{11}$ Estos sabores, aunque específicos, muestran la antigua fusión de saberes y sabores que se han producido en el Índico, y, desde este, con el resto del mundo

\footnotetext{
${ }^{9}$ Véase, a este respeto, las siguientes páginas web: http://www.independenciaslusa.info/receita-um-caril-decamarao-no-quintal/; http://www.mmo.co.mz/gastronomia/caril-de-camarao;

http://gnt.globo.com/receitas/receitas/caril-de-camarao.htm accedidas en 22 de agosto de 2016.

${ }^{10}$ Especie de tortilla hecha con harina de trigo.

${ }^{11}$ Conserva picante y acida, normalmente de verduras o frutas.
} 


\section{Revista \\ Debates Insubmissos}

(Meneses, 2009a). A este respecto, se puede considerar como uno de los precursores de la comida de fusión contemporánea.

En la confección del caril de hojas de maní, ${ }^{12}$ base de la alimentación de la mayoría de la población del sur de Mozambique, está las hojas, tomate y pimienta negra (a veces coco); este plato se sirve con arroz (o con maíz o mandioca), alimentos que se consumen tanto en las Américas como en el Índico. Estos alimentos reflejan los contactos y las apropiaciones culturales que empezaron hace más de 500 años (Meneses, 2016). Sin embargo, muchos de los estudios sobre la alimentación, la cocina e incluso la seguridad alimentaria insisten en separar las comunidades locales de los sistemas mundiales, haciendo hincapié en la lectura evolucionista y lineal de los procesos culturales.

La constitución, desde la modernidad eurocéntrica, de una jerarquía intelectual, impuso como canon las culturas del Norte global y sus tradiciones intelectuales, registros culturales que se auto-atribuyen un lugar de superioridad (Trouillot, 2002, p. 221-222). Este proceso supone la minimización del 'resto' periférico como símbolo de atraso, desde una perspectiva evolucionista sobre el mundo globalizado. ${ }^{13}$ Estos dos elementos autoconstrucción del centro y de la periferia - son momentos centrales del proyecto de la modernidad eurocéntrica, que produjeron como periférica la contribución de África, de Asia e de las Américas en la creación del proyecto político y epistemológico de la modernidad. ${ }^{14} \mathrm{O}$ sea, mucho del saber sobre los diversos contextos africanos entra en el canon del saber traducido desde una perspectiva epistemológica específica que genera 'universales de referencia' desde el Norte global. La dificultad sigue porque estos 'universales' no son únicamente referenciales; paradójicamente ellos ofrecen visiones del mundo que se intentan exportar como el saber más legítimo e importante. Y en estos universales producidos desde el Norte global, el continente africano emerge como espacio de hambre y pobreza permanente

\footnotetext{
${ }^{12} \mathrm{O}$ de mandioca, calabaza, frijoles, cacana (Momordica balsamina), entre otras hojas.

${ }^{13}$ Por ejemplo, el trabajo de Luis Vicente de Simoni sobre Mozambique (1821) refleja claramente una postura imperial en relación a la interpretación de la realidad observada.

${ }^{14}$ Este reto está en línea con las propuestas de Dipesh Chakrabarty (2008) y Boaventura de Sousa Santos (2010), para quienes el pensamiento europeo es indispensable pero insuficiente para comprender adecuadamente a las sociedades modernas víctimas de la violencia colonial, así como a Europa (esta misma con varios espacios subalternizados). Esta amnesia cronológica y el silenciamiento y ocultación de otras narrativas históricas impide, incluso, la comprensión del lugar del Norte global.
} 
(Devereux, 2007), con sistemas de producción alimentar retrasados, como la fundación Gates plantea. $^{15}$

La creación del eurocentrismo tras un espacio-tiempo concebido como excepcional y central forja el mundo colonial como un espacio externo y anterior al progreso de Europa (Bhabha, 1994, p. 250). Concretamente, los procesos coloniales y los crueles episodios de violencia asociados a estos son borrados, o silenciados, como que justificados por la marcha de la historia universal. Un ejemplo de esto son los trabajos de Fernand Braudel sobre la Civilización material, economía y capitalismo (1984). Las propuestas analíticas de Braudel pecan por dar seguimiento a una lectura kantiana de la historia, vista como un proceso espacio-temporal linear que iba de oriente a occidente. Pero esta historia debe ser reconceptualizada para que sea posible dialogar, por ejemplo, con los flujos comerciales internacionales en el Océano Índico desde muchos siglos atrás (Pearson, 2015).

Como esta síntesis señala, la globalización no fue un producto patentado por el Norte global. En el caso del Índico, Europa se juntó, especialmente desde el siglo XV, a los sistemas de intercambios comerciales que ya funcionaban desde hace mucho (Alpers, 2014). Estos contactos globales afirmaron episodios de progreso a partir de contactos marítimos, donde el intercambio de saberes y de culturas produjo ciudades cosmopolitas como El Cairo, Malabar, Zanzíbar, (isla de) Mozambique o Goa. En estos espacios, se hacían (y siguen haciéndose) transacciones de especias, sabores y saberes, donde los diálogos fermentaban más allá de la homogeneidad, el esencialismo o la conquista.

Estos y otros estudios realizados en varios contextos del Índico alertan para que las historias de contactos en este océano no sean leídas a través de las lentes planeadas por el Norte global. Los propios portugueses (y otros europeos) usaron las redes comerciales existentes en el circuito del Índico para obtener los productos deseados en Europa. O sea, se puede describir esta etapa inicial de la presencia europea en las costas orientales de África como un proceso de transición de un control comercial y de saberes por parte de diferentes grupos sociales del Índico a ser controlados por grupos europeos (Meneses, 2009a). El gran

\footnotetext{
${ }^{15}$ Véase https://www.gatesnotes.com/2015-annual-letter?lang=en\&page=0; https://www.gatesnotes.com/Development/Why-Does-Hunger-Still-Exist-Africas-Table-Day-One, Recuperado el 22 de agosto de 2016.
} 
cambio surge en la mitad del siglo XIX, con la ocupación efectiva del espacio que era Mozambique por Portugal imperial, introduciendo un sistema de administración política y científica que se tradujo en la implantación de la moderna colonización (Meneses, 2010).

Asociada al moderno proyecto colonial llega la idea de la tierra como mercancía, y de lo africano 'indígena' como ignorante e incapaz de producir (y garantizar la alimentación) para sí mismo y para los suyos. Desde entonces Mozambique, como muchos países de la región, ha conocido la introducción del monocultivo (azúcar, tabaco, arroz, etc.) con poco o ningún apoyo a la economía campesina que sustenta grande parte de los mozambiqueños. ${ }^{16} \mathrm{El}$ ciclo vicioso de las dependencias de ‘apoyos externos' (y de intervención en la selección de estrategias económicas nacionales) ha continuado con la independencia (en 1975), como afirman varios economistas (Mosca, 2010; Castel-Branco, Massingue y Muianga, 2015).

\section{OTRAS VOCES, OTROS SABERES}

A medida que avanzaba el siglo XX, el sur de Mozambique va a conocer la migración masculina hasta Sudáfrica, los impuestos, la producción de cultivos forzados y la crisis ecológica que va socavar la economía campesina, requiriendo de las mujeres el refuerzo de las solidaridades a través de redes afectivas y de condivisión de saberes asociados a la sobrevivencia y a los alimentos.

Fueron tiempos difíciles. Me quedé sin mi marido que se fue a las minas en Sudáfrica. Yo no era de Maputo, ${ }^{17}$ y no tenía familiares acá. Y él enviaba plata así de vez en cuando. Para lograr sobrevivir con los niños, para que fueran a la escuela, decidí abrir una 'machamba' (huerta) en las 'zonas verdes', ${ }^{18}$ donde producía para nosotros y también vendía a los vecinos. Tuve que pedir permiso para abrir el cultivo al régulo (la autoridad tradicional) y tendría de pagarle también. Pero como había aprendido de mi madre como hacer el cultivo, logramos sobrevivir; hasta ahora sigo produciendo en mi machamba. Fui aprendiendo a plantar otros productos que en mi región no crecen. Y así fuimos viviendo, aún cuando mi esposo regresó. Sigo siendo una campesina en la ciudad (Margarida Mungwambe, Maputo, 2004).

\footnotetext{
${ }^{16}$ Más de la mitad de la populación del país es campesina.

${ }^{17}$ Capital de Mozambique.

18 Región al redor de la ciudad de Maputo, donde muchas personas hacían cultivo agrícola para sí y para comercialización a pequeña escala.
} 


\section{Revista \\ Debates Insubmissos}

Esta realidad fue presentada por varias mujeres entrevistadas, exponiendo la necesidad de dar espacio a otras narrativas que desafíen los relatos que insisten en construir África y los africanos - en su diversidad - como espacio de desastres y hambre, rehenes de la tradición. Como Ranajit Guha señala, oír atentamente a las pequeñas voces silenciadas posibilita desafiar la univocidad de cualquier discurso, trayendo de regreso a la narrativa la cuestión de la agencia y de la instrumentalidad (1996, p. 11).

A ejemplo de Margarida, muchas mujeres en Mozambique son campesinas, aun viviendo en la ciudad. Este carácter hibrido de su identidad requiere que esta categoría social sea tratada en profundidad. La descripción de 'agricultura tradicional' es usada casi como sinónimo de formas de producción que anteceden la llegada de las 'modernas' técnicas agroindustriales e irrigación. Pero la historia reciente de la agricultura en el sur de Mozambique es una historia de mujeres, de sustentabilidad e innovación, de esfuerzo y solidaridad, una realidad que desafía la idea esencialista sobre una existencia ancestral, sin cambios, anterior a la llegada de la administración colonial (Young, 1977, p. 68).

Yo trabajaba como empleada doméstica donde una pareja de portugueses que tenían varios hijos. Se fueron de Mozambique cuando hubo la independencia. No lograron aceptar que fuéramos dueñas de nosotras mismas. A menudo me decían que íbamos a estar peor acá, después que los portugueses se hubieran ido. Y en verdad no fue fácil sobrevivir. No tenía trabajo, y tampoco mi esposo. La fábrica donde él trabajaba se quedó sin materiales para producir y no había dinero. Decidimos hacer una machamba para alimentarnos en la familia. Y seguimos trabajando. Producimos para nosotros y después mi esposo va a vender a las señoras que están en los mercados aquí en Maputo (Joana Manjate, Maputo, 2005).

El desarrollo de la producción agrícola campesina para generación de efectivos no resultó ni en la presencia de una agricultura atrasada, ni en la dominación masculina en la agricultura campesina contemporánea. Las mujeres han seguido desarrollando una producción para generación de efectivo, alargando el espacio de producción al redor de la ciudad de Maputo (Veronez de Sousa, 2014).

Cuando llegamos acá para abrir nuestra machamba, éramos varias mujeres. Cada una tenía su pedazo. Pero éramos de regiones diferentes acá, del sur. Y nos fuimos ayudando y ampliando nuestra producción. Al inicio era más 


\section{Revista \\ Debates Insubmissos}

mandioca y maíz, y después fuimos añadiendo otros alimentos, como maní, calabazas, tomates, pimentones, frijoles.... Yo no sabía cómo plantarlos y cuidarlos, pero una de las compañeras lo sabía, tendría apoyo de sus jefes que trabajaban en el ministerio [de agricultura], y así nos fuimos. Fuimos conociendo las mejores semillas, sobre el uso de fertilizantes. [...] ¡Nuestra producción ha mejorado mucho! (María Monjane, Maputo, 2002).

Todavía, como los casos analizados ilustran, el pensamiento científico moderno insiste en imponer una fractura abismal, dividiendo el mundo en un mundo moderno - el Norte global - relegando todas las 'otras' geografías a una categoría local, tradicional, sinónimo de atraso y de subdesarrollo, con saberes únicamente con valor local. Como Boaventura de Sousa Santos resalta, el poder de la colonialidad del saber es tan fuerte que las líneas abismales siguen, donde

el otro lado de la línea desaparece como realidad, se convierte en no existente $[. .$.$] . No existente significa no existir en ninguna forma relevante o$ comprensible de ser. Lo que es producido como no existente es radicalmente excluido porque se encuentra más allá del universo de lo que la concepción aceptada de inclusión considera que sea su otro. Fundamentalmente lo que más caracteriza el pensamiento abismal es pues la imposibilidad de la copresencia de los dos lados de la línea $(2010$, p.11).

Esta fractura, originada por la diferencia colonial, refleja un proyecto epistemológico hegemónico que demerita y excluye a todos los otros saberes constituidos fuera del alcance de la racionalidad científica moderna (Santos, Meneses y Nunes, 2005, p. 33-34). Como consecuencia, desde la perspectiva del Norte global, los 'otros' saberes producidos como noexistentes / excluidos de la racionalidad moderna, han producido no-seres subalternos, silenciados e incapaces, muchas veces, de traducir sus saberes al discurso del saber técnicocientífico, dominante.

Nosotras, campesinas, hemos escuchado sobre la soberanía alimentaria. Lo entendemos porque somos de acá, tendremos derecho a usar esta tierra donde vivimos y trabajamos, donde hemos alimentado nuestras familias. La tierra no es solamente de los hombres, es nuestra también. Fue de nuestras manos que producimos nuestra propia comida [...]. Trabajamos la tierra con lo que conocimos, con la experiencia que hemos aprendido desde nuestras madres [conocimientos locales...], en consulta con lo que los técnicos nos aportan [conocimientos científicos]. Trabajamos juntos, con el apoyo de nuestros familiares, y fue así que con nuestro trabajo hemos llegado hasta donde estamos, que nuestros esposos nos respectan por nuestra posición acá 


\section{Revista (O) \\ Debates Insubmissos}

[en la comunidad]. Mira nuestros hijos. Ahora son adultos, pero son fuertes! Esta es nuestra soberanía, poder sostener nuestra familia, la comunidad, desde lo que producimos de nuestras manos (Francisca Arone, Maputo, 2010).

Las mujeres están atribuyendo un valor específico a los alimentos, valor que no es reducible a las cualidades nutricionales científicamente evaluables o a las cantidades de alimentos producidos por o administrados a grupos particulares. Las perspectivas que destacan solamente la producción de alimentos, mano de obra, preparación y consumo, pierden de perspectiva la estrecha interrelación de estos elementos a la posesión de la tierra, al camino de una comunidad de vida, los inter-conocimientos, los regalos recíprocos y sustento de vida, elementos que conectan a las personas en una comunidad, y el respeto a la vida no humana.

Las mujeres, con especial preponderancia en las últimas décadas, vienen cuestionado su lugar de saber, ser y poder a partir de sus propias experiencias, desafiando la centralidad y la legitimidad singular del conocimiento moderno, muchas veces promovido como política central por el Estado. Este es el caso de los debates actuales sobre las políticas agrícolas en Mozambique, donde la apuesta reciente parece insistir en la sustitución de los conocimientos locales, tradicionales, por la experiencia agrícola 'más avanzada' de los monocultivos en el marco de la agricultura industrial. Y con esta opción política del gobierno, una parte importante de la población sigue siendo considerada ciudadana sin voz ni saberes, es decir, sigue manteniéndose en una condición de subhumanidad, cuyos intereses no son tenidos en cuenta en la construcción de las políticas nacionales.

Un ejemplo de esta opción es el proyecto ProSAVANA que tuvo inicio en Mozambique en 2011, fruto de la cooperación institucional entre Mozambique, Brasil y Japón. Sus objetivos son la exportación de tecnología brasileña para mejorar el desarrollo agrícola y rural en Mozambique, aumentar la producción y competitividad del sector en el ámbito de la seguridad alimentaria y la generación de excedentes exportables. ${ }^{19}$ Para las campesinas y campesinos, lo que este proyecto significa es la pérdida de la tierra para más de

\footnotetext{
${ }^{19}$ Ver http://www.prosavana.gov.mz/.
} 
4 millones de personas en el país, cuyas prácticas agrícolas se ven como poco productivas porque contribuyen poco a la producción de cereales y otros productos para la exportación.

Desafiando esta propuesta del gobierno de Mozambique, varias asociaciones de campesinos han iniciado un proceso de cuestionamiento de las intenciones reales de ProSAVANA, criticándolo por ser consecuencia de una política hecha desde arriba hacia abajo y que no tiene en cuenta las demandas, sueños y preocupaciones básicas de los campesinos. ${ }^{20}$ En la opinión de varios movimientos sociales, ProSAVANA dará lugar a "la aparición de comunidades sin tierra, a la agitación social, a la pobreza, a la corrupción y a la destrucción del medio ambiente" (UNAC - Via Campesina Africa - Grain, 2012). Las voces críticas han levantado varias cuestiones sobre potenciales peligros sociales y ambientales de la agricultura de monocultivo, los peligros de animar a los agricultores a utilizar semillas genéticamente modificadas, y la probabilidad de que los insumos de alto costo darán lugar al aumento de las desigualdades dentro de las comunidades agrícolas africanas. ¿Es realmente una buena idea, los críticos se preguntan, que los agricultores africanos sean más dependientes de insumos extranjeros proporcionados por las empresas multinacionales?

En una reunión promocionada por diversos movimientos sociales en 2013, se ha producido una "Carta Abierta para Detener y Reflexionar de Manera Urgente el Programa ProSAVANA" donde se exigió que "todos los recursos humanos, materiales y financieros asignados al programa ProSAVANA sean reubicados en la definición y la implementación de un plan Nacional de Apoyo a la Agricultura Familiar Sostenible que se ha abogado por más de dos décadas por las familias campesinas de Mozambique". ${ }^{21}$

Como esta lucha puntea, la permanencia de una fractura producida por la diferencia colonial abismal oculta la persistencia de relaciones e interpretaciones coloniales tanto en el nivel

20 Declaración inaugural de la Campaña Não ao ProSAVANA! (junio 2014) disponible en http://farmlandgrab.org/23578. Las denuncias críticas del ProSAVANA se iniciarán en octubre de 2012 (http://www.unac.org.mz/index.php/7-blog/39-pronunciamento-da-unac-sobre-o-programa-prosavana;

http://farmlandgrab.org/21566). Desde entonces varias declaraciones de apoyo han sido divulgadas por movimientos sociales de Brasil, Mozambique y Japón, así como por organizaciones internacionales. Véase http://www.farmlandgrab.org/cat/show/827, accedido el 26 de agosto de 2016.

${ }^{21}$ Disponible en http://alice.ces.uc.pt/news/?p=3391, accedido el 21 de Agosto de 2016. Véase igualmente el "Comunicado conjunto e questionamentos da sociedade civil de Moçambique, Brasil e Japão sobre o ProSAVANA com relação aos documentos do governo recentemente vazados” (27 de Agosto de 2016), disponible en http://www.farmlandgrab.org/post/view/26458. 
epistémico (los 'otros' no saben cómo pensar) como en el nivel ontológico (los 'otros' existen, pero no cuentan): los campesinos y las campesinas no tienen saberes y experiencias válidas (a la luz de la métrica económico capitalista) y no cuentan como sujetos políticos activos.

La consciencia activa de las campesinas y el apoyo y solidaridad de movimientos nacionales e internacionales ${ }^{22}$ reflejan la emergencia de una dimensión política en juego: contrario al concepto más bien técnico de la seguridad alimentaria, los argumentos de la soberanía alimentaria subrayan las responsabilidades de las personas y de los gobiernos, señalando la necesidad de tener en cuenta las consecuencias locales de procesos políticos y económicos macro.

A pesar de los numerosos episodios de silenciamiento y supresión de historias y saberes, el mundo contemporáneo sigue presentando una enorme diversidad de procesos que confieren inteligibilidad e intencionalidad a las experiencias sociales. Como intentaré debatir en el punto siguiente, es en este Sur global donde numerosas epistemologías rivalizan, se interpenetran y dialogan entre sí (Santos y Meneses, 2014, p. 8-9).

\section{LA COCINA COMO LABORATORIO DE LAS EPISTEMOLOGÍAS DEL SUR}

En los últimos años son numerosos los trabajos que buscan dar cuenta de otras voces y saberes en el Sur global, donde el reconocimiento por la centralidad de las mujeres está en aumento (Bose y Kim, 2009), recentrando otras formas de experimentar y sentir el mundo, como el olor o el sabor.

La intensificación, a partir de las revoluciones burguesas europeas, especialmente desde el siglo XVIII, de la centralidad de una corporalidad que se aleja de su componente natural, va a erigir una tríada civilizacional - higiene, orden y belleza (Laporte, 2000, p. 84-85). En esta tríade, producto de un ambiente sexista y colonial, los olores y sabores otros son vistos como sospechosos y atribuidos a pueblos inferiores, relegados así a una situación de alteridad subalterna. Esta subalternización por las epistemologías del norte se explica probablemente porque amenazan el régimen abstracto de la modernidad eurocéntrica, basada en la

\footnotetext{
${ }^{22}$ De derechos humanos, ambientales, feministas, ecológicos, etc.
} 
información captada por la visión y la audición. Como buscaré teorizar, centrar nuestra racionalidad en el sentido de la vista contribuye al mantenimiento de las estructuras de silenciamiento de los saberes y luchas de las mujeres, impidiendo la ampliación a otros imaginarios acerca de los saberes del mundo.

En este contexto, poner el foco en las mujeres y la cocina del Sur global es particularmente importante, ya que las políticas de sexo y género han jugado un papel central en la construcción y en la legitimación de las relaciones económicas, culturales, y epistémicas que continúan dominando las relaciones de poder en el mundo. Hay que cuestionar el proyecto colonial-capitalista y patriarcal contemporáneo, y las jerarquías de saber y poder que lo conforman y que siguen produciendo seres subalternizados, invisibilizados y silenciados. Pero es posible rescatar otros seres, cuyos saberes apuntan hacia otras historias de contactos e hibridaciones de saberes (Meneses, 2009a, 2016). Es central a este rescate el abordaje que ofrecen las epistemologías del sur.

Como señala Boaventura de Sousa Santos (2014), una de las ideas centrales de las epistemologías del sur es que la comprensión del mundo excede en mucho la comprensión eurocéntrica del mundo. La racionalidad estético-expresiva eurocéntrica no solo no da cuenta de la diversidad de saberes en el mundo, sino que además contribuye, al sustentarse en una interpretación parcial del mundo, a eliminar otras narrativas y teorizaciones acerca de la diversidad en que vivimos. La racionalidad de las epistemologías del sur sucede cuando convivimos comiendo, compartiendo sabores, sazonando los diálogos entre saberes. Las razones que experimentan las emociones implican otra cultura del cuerpo y de las emociones. En ese sentido exigen reflexionar desde otra forma de ser y experimentar el mundo.

Preparar un plato es aprender desde los laboratorios que son las cocinas. En Mozambique, así como en otros lugares del mundo, la cocina es performativa: en ella participan tanto la cocinera, como la familia, amigos y otros invitados, comiendo, comentando y apreciando el acto. La cocina es, en este contexto, dominio de las mujeres, que controlan estos laboratorios donde se combinan saberes especializados (Meneses, 2016). 


\section{nevistat \\ Debates Insubmissos}

En las palabras de la señora Luisa, que me enseñaba como hacer un caril de maní, ${ }^{23}$

Siempre he estado preparando este caril desde que recuerdo, y yo empecé a prepararlo ayudando a mi madre. [...] La forma en que hago el guiso, es de hecho la receta de mi familia. Acá preparamos el caril de una manera diferente. Yo no uso coco y si maní machacado. Y prefiero usar los camarones secos al pollo. Pero se puede también agregar pollo si a usted le gusta. ¡Usted tiene que tener tiempo, esto no es un plato de esos modernos, que se cocina en un par de minutos!

La preparación varía, dependiendo de la persona que cocina. Algunos cocineros apilan los ingredientes en niveles - la cebolla, el tomate.... Otros cocinan primero un salteado hecho con cebollas y tomates, salteados en aceite vegetal, ajo y pimienta. Hay que agregar entonces la leche de maní y después, lentamente, los otros ingredientes. Porque el caril se está cocinando en la leche de maní... Nosotras del sur más bien utilizamos la leche de maní en lugar de la leche de coco. ¿Sabe usted hacer la leche? ¡Es que vi algunas personas intentando hacerlo por ahí y dejan mucho que desear! caril de maní que sea caril de maní tiene que ser de maní machacados en un mortero y no molidos. Y no vale dejarlos casi un siglo en remojo en agua. ¿La leche espesa es fundamental para la salsa! El plato tiene que cocinar lentamente porque tiene que dejar que los sabores se combinen. [...] Este es un plato que sirve a cualquier situación. Se es rica, lo puede hacer con más productos, como pollo, cangrejo. Y nosotras, mujeres que tenemos nuestras machambas, cocinamos lo que producimos. El maní es de mi cultivo...

Conocer una sociedad a través de los sabores permite desafiar radicalmente la subalternidad de las mujeres y de sus formas de ser y estar, en el mundo, en la lucha. Moviéndose a través de varias culturas, los sabores y los saberes implicados en la preparación y consumo de alimentos se pueden interpretar como "zonas de contacto" (Pratt, 2002, p. 4), espacios de creatividad, de confrontación y de fusión, donde se articulan diferentes relaciones de poder entre formas de ser y de conocer.

¿Creé usted que haya mozambiqueña a la que no le gusta pilar en un mortero? En cualquier parte del país el pilar es sagrado... ah... la modernidad... ¡Lo cocino a este caril así desde hace muchos años... y siempre con el mortero! [...]

Antes, en lo tiempo de los portugueses [antes de la independencia] pocos eran los blancos que comían este caril. Esto era una comida de indígena [de los negros], como se decía entonces. Esta es la memoria que tienen acerca del caril. Los pocos blancos que de vez en cuando comían caril con nosotros tenían miedo de comidas extrañas, muy olorosas y picantes. Y tendríamos

\footnotetext{
${ }^{23}$ Este plato de la culinaria de Mozambique es muy conocido y tiene incluso, una página web sobre su preparación. Vea-se https://pt.wikipedia.org/wiki/Caril de amendoim, accedido en 21 de agosto de 2016.
} 


\section{Revista \\ Debates Insubmissos}

que comerlo con cucharas si ellos estaban presentes. Ya que nosotros comemos el caril con arroz o chima $^{24}$ con nuestras manos, y las manos pueden quedarse grasosas. Hay que saber comer. Pero entonces comer en público con las manos era un símbolo de ser indígena, no civilizado. ¡Hoy en día se puede comer muchas formas diferentes del caril, incluso en los hoteles! (Luisa Macombe, Maputo, 2012).

Como otras formas de performance oral que combinan la creatividad con la consistencia de la reproducción, la erudición en la cocina se produce tanto por memorias individuales, como de grupos que compartían una cocina, pero solo muy recientemente de forma escrita (McCann, 2009). En Mozambique, como en otros países, la cocina se sostiene en una gramática de saberes que se exprime a través de la oralidad, articulando experiencia, práctica y replicabilidad. Las prácticas de las cocineras expresan las historias y los encuentros de las culturas, reflejando opciones y experiencias políticas: por ejemplo, las innovaciones técnicas en la adaptación a la cultura y el procesamiento de nuevos alimentos (maíz, maní, ají, mandioca o fríjoles, todos oriundos de las Américas), de bienes comercializados (semillas o sal) o de ingredientes perecederos prestados de sus vecinos (hojas, frutos, especias). Del mismo modo, las relaciones que generan desigualdades y dominación también están expresadas en la cultura de la cocina. Las pastas y el pan, por ejemplo, aparecen en las ciudades coloniales del África austral con la implantación de la colonización europea moderna (Meneses, 2009a).

Cocinar es una forma de saber y de ser, de expresar emociones. La receta encierra la historia de los ingredientes, de su búsqueda y uso en la preparación, los estilos de cocinar y los modos de presentar y consumir los alimentos. La cocina africana, en su diversidad, muestra las culturas del continente y refleja, en paralelo, los encuentros y contactos con regiones vecinas, desafiando las ideas acerca de un centro y una periferia. Muchas de las recetas y de los platos que las mujeres negras de las Américas confeccionan contienen información antigua sobre la preparación de diferentes platos de diversas regiones del continente africano (Clark, 2007), y vice versa. Como dijo una de las mujeres entrevistadas: “¿Usted dice que el maní viene de América? Pude ser, pero eso fue hace muchos años. Ahora

\footnotetext{
${ }^{24}$ Pasta hecha con harina de maíz.
} 
es nuestro, de Mozambique. Y sabemos muy bien como cocinarlo" (María Monjane, Maputo, 2002).

Esos saberes africanos, que contienen en sí saberes de otros lugares del mundo, tras contactos milenarios, atravesaron el Índico y el Atlántico en la carga de los navíos, bien sea de la mano de comerciantes, o sea de personas traficadas como esclavas durante más de 300 años. En este caminar, las mujeres africanas ajustaron y transformaron sus recetas para sustituir parte de los productos que no encontraban en esta parte del mundo, usando productos, utensilios y métodos locales desconocidos, junto con ingredientes y abordajes oriundos de Europa, Américas y de Asia. En conjunto, estas mujeres contribuirían a la reproducción social de sus grupos, mismo en condiciones extremas.

\section{CONCLUSIONES}

Las recetas, muchas de ellas circulando apenas en el campo de la oralidad, dicen mucho acerca de encuentros de saberes y de culturas, acerca de las historias de estas mujeres como sujetos de sus procesos identitarios, un conjunto poderoso de pequeñas voces que expresan su saber, su forma de ser. ¿Qué hacer para que estas narrativas históricas, escritas y orales, sean vistas como archivos que conservan los nexos epistemológicos y estéticos entre las mujeres del continente y las de la diáspora (Clark, 2007: 151)? Desde la perspectiva del cosmopolitismo subalterno (Santos, 2010, p. 30), ${ }^{25}$ la llamada es para que se miren estas recetas - parte central de las trayectorias de vida de estas mujeres - como un registro importante de saberes, que permite recuperar la estética de generaciones de mujeres desconocidas, cuya arte se manifestó en sus jardines, huertas y cocinas. La cocina trata de los sabores, de los ciclos de la vida que se han ignorado y convertido en periféricos durante siglos, combinando dos no-seres: las mujeres y la epistemología de los sabores.

Las recetas son una forma en que las mujeres quienes cargan a sus espaldas la 'carga de la colonialidad' reclaman sus luchas y recrean sus historias, produciendo un archivo de

\footnotetext{
${ }^{25}$ Para Santos, se trata de un "conjunto extenso de redes, iniciativas, organizaciones y movimientos que luchan contra la exclusión económica, social, política y cultural generada por la encarnación más reciente del capitalismo global, conocida como globalización neoliberal” (2010, p. 30).
} 


\section{Revista \\ Debates Insubmissos}

epistemologías de mujeres unidas a través de los circuitos de la violencia colonial, del comercio y de la diáspora. Y la función del archivo es precisamente la de facilitar la recuperación de las historias de los no-seres que confrontaron numerosas tentativas de aniquilación, en actos de genocidio, esclavitud, patriarcado, colonización y erradicación de las memorias culturales. Incluyendo a las mujeres como sujetos en la plenitud de los campos en los que ellas actúan aumentamos la posibilidad de desafiar la hegemonía del saber científico moderno.

Cuando se estudia la diversidad de realidades alimentares por todo el mundo es posible identificar alternativas locales, pero son utopías concretas de innovación y sustentabilidad que se están realizando. El reto es darles credibilidad, darlas a conocer cada vez más. Tras las epistemologías del sur es posible desarrollar un pensamiento alternativo de alternativas (Santos, 2004; 2014) para que podamos dar credibilidad a todo ese saber tan diverso, descolonizando la imaginación, a través de la justicia cognitiva (Meneses, 2009b, p. 233-234).

Superar la situación de colonización epistémica exige, como Boaventura de Sousa Santos subraya (2010), que se acepte el desafío de ir para el Sur y aprender con el Sur, no como Sur imperial (que reproduce en el Sur la lógica del Norte, asumida como universal); al contrario, es preciso aprender del Sur anti-imperial, en su diversidad, más allá de la biblioteca colonial.

Dar voz a las subalternas, aprender a escucharlas y traducirlas interculturalmente no es escribir, de nuevo, narrativas que se consagran en un 'nuevo centro', en un nuevo canon; de esta manera se esencializa la diferencia de forma radical, imposibilitando cualquier traducción intercultural. Al contrario, la búsqueda de las especificidades de los procesos, dar voz y aprender a escuchar las voces silenciadas por su diferencia radical, asume una importancia crucial en este proceso de traducción, una forma de no caer en las trampas generadas por el indigenismo o el esencialismo. Reconocer los saberes asociados a la producción y consumo sostenibles, en varios contextos del mondo, requiere que se reconozca la traducción e intercambio cultural, entre saberes y sabores. En las palabras de Boaventura de Sousa Santos, la traducción intercultural consiste en buscar intereses isomorfos y supuesto subyacentes entre 
las culturas, identificando diferencias y similitudes (2014, p. 212). De esta forma es posible reconocer que cualquier cultura es dinámica y que comunica a menudo con otras culturas, una respuesta a un universalismo abstracto eurocéntrico que representa como locales y inconmensurables los múltiples saberes de Sul global.

Como intenté problematizar en este texto, la sostenibilidad es un concepto sistémico, que pasa por un conocimiento profundo de la relación de continuidad entre los aspectos económicos, sociales, culturales y ambientales de cada sociedad. Discutir la soberanía alimentaria respetando la sociobiodiversidad implica una comprensión más amplia de contextos históricos y humanos, a través de las biografías de las recetas y de las prácticas asociadas. El uso de ingredientes de producción local es la base de lo que ahora se llama la cocina sostenible o "del jardín a la mesa". Este recurso ha sido amplificado por varios chefs en el mundo, que afirman "hacerse conscientes de lo insostenible sistema alimentario global" ${ }^{26}$ que deja a millones con hambre y en la pobreza; esta posición sigue (re)produciendo una lógica colonial de poder y de saber que no reconoce la experiencia y la sabiduría presentes en el mundo sobre qué comer y cómo comer. Superar estas situaciones de injusticia alimentaria implica necesariamente el cambio radical de la relación dicotómica sujeto / objeto, estrechamente asociada con la construcción jerárquica del conocimiento y a su separación.

Como he plateado, la justicia social global no es posible sin la justicia cognitiva (Meneses, 2009b, p. 235). El análisis cuidadoso de la economía política asociada a la alimentación en Mozambique requiere que la producción, el consumo y la valorización de los saberes sea hecha desde abajo, de manera popular y pública, convirtiéndose en la base de la comprensión de la auto-definición y de la libre determinación de las comunidades. Esta propuesta desafía las diferencias abismales, lo que requiere que sean producidas epistemologías que respeten y representen a la diferencia según las experiencias de lucha, evitando las trampas de los estudios superficiales y poco críticos sobre estas diferencias. Es una propuesta epistémica que incluye no sólo los derechos de los científicos disidentes dentro de un paradigma dominante, sino también los derechos de las epistemologías alternativas y de los saberes alternativos (Visvanathan, 2003, p. 729). Como nueva gramática global, contra-

\footnotetext{
${ }^{26}$ Véase, por ejemplo, la página https://www.globalcitizen.org/en/content/10-eco-chefs-blending-culture-andsustainability-i/, accedida el 20 de Agosto de 2016.
} 
hegemónica, la justicia cognitiva exige, sobre todo, la necesidad urgente de dar visibilidad y espacio de acción a otras formas de conocer y experimentar el mundo, especialmente de los saberes marginados y subalternizados (Meneses, 2009b, p. 237). Esta propuesta epistémica también es ontológica, es decir, busca recuperar otros conocimientos, (re)visitar otros circuitos, (re)adquirir otros sentidos. En este contexto, traer a lo debate los saberes de otras culturas representa un deber doble: ampliar las posibilidades y condiciones de (re)conocimiento de múltiples sentidos de la dignidad humana, de los saberes y sabores de diferentes comunidades, grupos, y por otro, (re)aprender a establecer y profundizar la intertraducibilidad entre esos saberes, en su diversidad, parte de culturas dinámicas de nuestro mundo.

\section{REFERÊNCIAS}

ALPERS, Edward.The Indian Ocean in World History. Oxford: Oxford University Press, 2014.

APPADURAI, Arjun. "How to Make a National Cuisine: cookbooks in contemporary India", Contemporary Studies in Society and History, 30 (1): 3-24, 1988.

. La vida social de las cosas. México, DF: Consejo Nacional para la Cultura y las

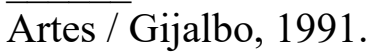

BHABHA, Homi K.. The Location of Culture. London: Routledge, 1994.

BOSE, Christine E.; KIM, Minjeong (ed.). Global Gender Research: Transnational perspectives. New York: Routledge, 2009.

BRAUDEL, Fernand. Civilización Material, Economía y Capitalismo. Siglos XV-XVIII (3 volumes). Madrid: Alianza Editorial, 1984.

CASTELO-BRANCO, Carlos Nuno; MASSINGUE, Nelsa; MUIANGA, Carlos (eds.) Questões sobre o desenvolvimento produtivo em Moçambique. Maputo IESE, 2015.

CHAKRABARTY, Dipesh. Al margen de Europa: pensamiento poscolonial y diferencia histórica. Barcelona: Tusquets, 2008. 
CLARK, Patricia E. "Archiving Epistemologies and the Narrativity of Recipes in Ntozake Shange's 'Sassafrass, Cypress \& Indigo””, Callaloo, 30 (1): 150-162, 2007.

COMAROFF, Jean; COMAROFF, John. "Etnografia e imaginação histórica", Proa - Revista de Antropologia e Arte [on-line], 1 (2), disponível em https://dash.harvard.edu/bitstream/handle/1/12108657/comaroff.pdf? sequence=3. 2010.

DE SIMONI, Luis Vicente. Tratado Médico sobre Clima e Enfermidades de Moçambique. Lisboa: Biblioteca Nacional, cód. 1-26-18-22, 1981.

DEVEREUX, Stephen. "Why does famine persist in Africa?", Food Security, 1 (1): 25-35, 2009.

FERGUSON, Priscilla P."A cultural field in the making: gastronomy in 19th century France”, American Journal of Sociology, 104 (3): 597-641., 1998.

FOUCAULT, Michel. Les mots et les choses. Paris: Gallimar, 1966.

GUHA, Ranajit. History and the Limit of World-history. New York: Columbia University Press, 2002.

HAMILTON, Cherie Y. Cuisines of Portuguese Encounters. New York: Hippocrene Books, 2008.

INGLIS, David; GIMLIN, Debra (ed.). The Globalization of Food. Oxford: Berg, 2009.

INGOLD, Tim. Being Alive. Essays on movement, knowledge and description. London: Routledge, 2011

KONETZKE, Richard. El imperio español. Madrid: Ediciones Nueva Época,1946.

LAPORTE, Dominique. History of Shit. Cambridge, MA: MIT press, 2000.

LA VÍA CAMPESINA. The right to produce and access to land. Position of La Vía Campesina on Food Sovereignty presented at the World Food Summit, 13-17 November, Rome, 1996.

LEONG-SALOBIR, Cecilia. Food Culture in Colonial Asia. London: Routledge, 2011.

MALDONADO-TORRES, Nelson. "On the coloniality of being", Cultural Studies, 21 (2): 240-270, 2007.

MATAVEL, Nilza et al. Os Senhores da Terra - Análise Preliminar do Fenómeno de Usurpação de Terra em Moçambique. Maputo: Justiça Ambiental e UNAC, 2011. 
MCCANN, James C. Stirring the Pot: a history of African cuisine. Athens: Ohio University Press, 2009.

MENESES, Maria Paula. "Food, Recipes and Commodities of Empires: Mozambique in the Indian Ocean Network", Oficina do CES n 335, 2009a

. "Justiça Cognitiva", in Cattani, António et al. (ed.), Dicionário Internacional da Outra Economia. Coimbra: CES/Almedina, 231-236, 2009b

. 'O 'indígena' africano e o colono 'europeu': a construção da diferença por processos legais”, E-cadernos CES [Online], 7: 68-93, 2010.

"Ampliando las epistemologías del sur a partir de los sabores: diálogos desde los saberes de las mujeres de Mozambique", [en línea] Revista Andaluza de Antropología, 10: 10-28, 2016.

MINTZ, Sidney W. "Comida e antropologia: uma breve revisão", Revista Brasileira de Ciências Sociais, 16 (47): 30-41, 2001.

MINTZ, Sidney W.; DU BOIS, Christine M. "The Anthropology of Food and Eating", Annual Review of Anthropology, 31: 99-1 19, 2002.

MOSCA, João. Politicas Agrárias de (em) Moçambique (1975-2009). Lisboa: Escolar Editora, 2010.

PEARSON, Michael (ed.). Trade, circulation, and flow in the Indian Ocean World. London: Palgrave MacMillan, 2015.

PRATT, Marie-Louise. Imperial Eyes: travel writing and transculturation. New York: Routledge, 1992.

ROLETTA, Paola. Cozinha Tradicional de Mozambique. Lisboa: Europa-América, 2004.

ROLINGHER, Louise. Edible Identities: food, cultural mixing and the making of identities on the Swahili Coast. Edmonton: PhD Dissertation, University of Alberta, 2009.

SAMPAIO, Maria Fernanda. Sabores do Índico: receitas da cozinha moçambicana. Lisboa: Assírio \& Alvim, 2007.

SANTOS, Boaventura de Sousa. "The Fall of the Angelus Novus: beyond the modern game of roots and options", Current Sociology, 46 (2): 81-118, 1998.

"A Critique of Lazy Reason: against the waste of experience", in Wallenstein, Immanuel (ed.), The Modern World-System in the Long Durée. Boulder, CO: Paradigm, 157-197, 2004. 
. Para descolonizar el Occidente. Buenos Aires: CLACSO / Prometeo Libros, 2010.

2014.

Epistemologies of the South. Justice against Epistemicide. Boulder: Paradigm,

SANTOS, Boaventura de Sousa; MENESES, Maria Paula; NUNES, João Arriscado. "Introdução. Para ampliar o cânone da ciência: a diversidade epistémica do mundo", in Santos, Boaventura de Sousa (ed.), Semear outras soluções: os caminhos da biodiversidade e dos conhecimentos rivais. Rio de Janeiro: Civilização Brasileira, 25-68, 2005.

SANTOS, Boaventura de Sousa; MENESES, María Paula. "Introdução", in Santos, Boaventura de Sousa; Meneses, Maria Paula (ed.), Epistemologias del Sur. Madrid: Akal, 7$17,2014$.

SHERIFF, Abdul. Slaves, Spices and Ivory in Zanzibar. London: James Currey, 1987.

TROUILLOT, Michel-Rolph. "The Otherwise Modern. Caribbean Lessons from the Savage Slot", in Knauft, Bruce M. (ed.), Critically Modern: alternatives, alterities, anthropologies. Bloomington: Indiana University Press, 220-237, 2002.

UNAC - Via Campesina Africa - GRAIN (2012), Mega projeto brasileiro poderá deslocar milhões de camponeses em Moçambique. Disponível em https://www.grain.org/article/entries/4628-mega-projeto-brasileiro-podera-deslocar-milhoesde-camponeses-em-mocambique, acedido a 22 de Agosto de 2016.

VERONEZ DE SOUSA, Leonardo. Experiências de agricultura (peri)urbana coletiva: outras experiências económicas? Coimbra: Tese de Doutoramento, Universidade de Coimbra, 2014.

VISVANATHAN, Shiv. "Convite para uma Guerra da Ciência", in Santos, Boaventura de Sousa (ed.), Conhecimento prudente para uma vida decente: 'Um discurso sobre a ciência' revisitado. Porto: Afrontamento, 717-734, 2003.

YOUNG, Sherilynn. "Fertility and Famine: Women's Agricultural History in Southern Mozambique," in Palmer, Robin; Parsons, Neil (eds.), The Roots of Rural Poverty in Southern Africa. Berkeley: University of California Press, 68-81, 1977.

Submetido em: 21/06/2018

Aprovado em: 25/06/2018 